(c) by Oldenbourg Wissenschaftsverlag, München

\title{
Crystal structure of tetracesium decaselenidotetrasilicate, $\mathrm{Cs}_{4} \mathrm{Si}_{4} \mathrm{Se}_{10}$
}

\author{
P. L. Feng, B. C. Chan and P. K. Dorhout* \\ Colorado State University, Department of Chemistry, Fort Collins, CO 80523, USA
}

Received December 21, 2004, accepted and available on-line January 21, 2005; CSD no. 409816

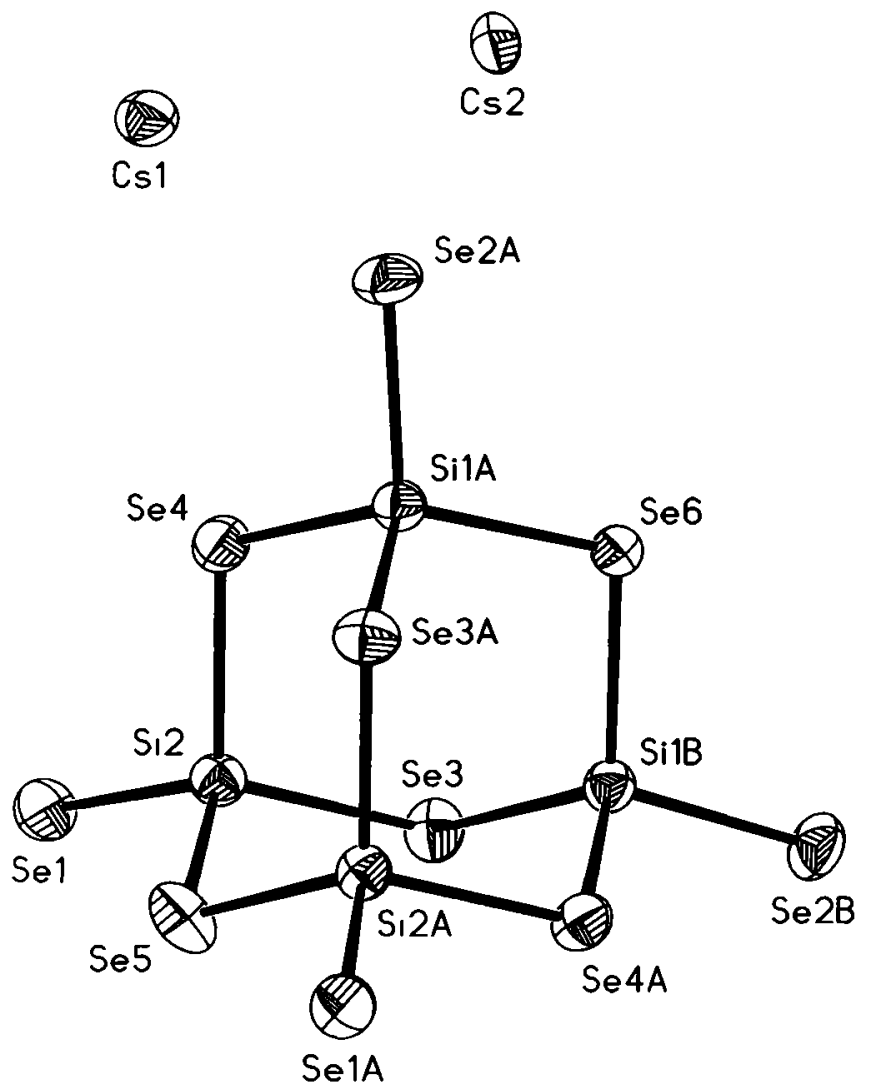

\section{Source of material}

Crystals of $\mathrm{Cs}_{4} \mathrm{Si}_{4} \mathrm{Se}_{10}$ were formed from a molten chalcogenide flux reaction of $127.12 \mathrm{mg} \mathrm{Cs} 2 \mathrm{Se}_{2}, 4.21 \mathrm{mg} \mathrm{Si}$, and $71.06 \mathrm{mg} \mathrm{Se}$. The reactants were combined in a fused silica ampoule in an inert atmosphere glovebox, sealed under vacuum, and heated to $750^{\circ} \mathrm{C}$ at a rate of $30 \mathrm{~K} /$ hour. After 200 hours of heating, the ampoule was cooled at $3 \mathrm{~K}$ /hour to room temperature. Dimethylformamide was added to dissolve remaining cesium selenide flux, resulting in well-formed colorless crystals of $\mathrm{Cs}_{4} \mathrm{Si}_{4} \mathrm{Se}_{10}$.

\section{Discussion}

$\mathrm{Cs}_{4} \mathrm{Si}_{4} \mathrm{Se}_{10}$ crystallizes with adamantane-like $\left[\mathrm{Si}_{4} \mathrm{Se}_{10}\right]^{4-}$ units composed of four corner-shared $\mathrm{SiSe}_{4}$ tetrahedra and $\mathrm{Cs}^{+}$cations for charge balancing the compound. $\mathrm{C}_{4} \mathrm{Si}_{4} \mathrm{Se}_{10}$ is isostructural to $\mathrm{Na}_{4} \mathrm{Ge}_{4} \mathrm{Se}_{10}$ [1], $\mathrm{Cs}_{4} \mathrm{Ge}_{4} \mathrm{~S}_{10}[2]$ and $\mathrm{K}_{4} \mathrm{Ge}_{4} \mathrm{Se}_{10}[3,4]$.

Table 1. Data collection and handling.

\begin{tabular}{ll}
\hline Crystal: & colorless block, \\
& size $0.03 \times 0.05 \times 0.08 \mathrm{~mm}$ \\
Wavelength: & Mo $K_{\alpha}$ radiation $(0.71073 \AA)$ \\
$\mu:$ & $208.90 \mathrm{~cm}^{-1}$ \\
Diffractometer, scan mode: & Bruker SMART CCD, $\varphi / \omega$ \\
$2 \theta_{\max }:$ & $56.56^{\circ}$ \\
$N(h k l)_{\text {measured, }} N(\boldsymbol{h k l})_{\text {unique: }}:$ & 11334,3012 \\
Criterion for $I_{\mathrm{obs}}, N(h k l)_{\mathrm{gt}}:$ & $I_{\text {obs }}>2 \sigma\left(I_{\mathrm{obs}}\right), 2115$ \\
$N($ param hefined: & 83 \\
Program: & SHELXTL [5] \\
\hline
\end{tabular}

Abstract

$\mathrm{Cs}_{4} \mathrm{Se}_{10} \mathrm{Si}_{4}$, monoclinic, $C 12 / c 1$ (no. 15), $a=16.164(2) \AA$, $b=16.314(2) \AA, c=9.723(1) \AA, \beta=106.683(3)^{\circ}$,

$V=2456.1 \AA^{3}, Z=4, R_{\mathrm{gt}}(F)=0.036, w R_{\text {ref }}\left(F^{2}\right)=0.083$,

$T=298 \mathrm{~K}$.

Table 2. Atomic coordinates and displacement parameters (in $\AA^{2}$ ).

\begin{tabular}{|c|c|c|c|c|c|c|c|c|c|c|}
\hline Atom & Site & $x$ & $y$ & $z$ & $U_{11}$ & $U_{22}$ & $U_{33}$ & $U_{12}$ & $U_{13}$ & $U_{23}$ \\
\hline $\mathrm{Cs}(1)$ & $8 f$ & $0.15246(3)$ & $0.13588(3)$ & $0.37597(5)$ & $0.0433(3)$ & $0.0435(3)$ & $0.0377(3)$ & $0.0104(2)$ & $0.0080(2)$ & $-0.0012(2)$ \\
\hline $\operatorname{Cs}(2)$ & $8 f$ & $0.36471(3)$ & $0.07139(3)$ & $0.85764(5)$ & $0.0408(3)$ & $0.0472(3)$ & $0.0399(3)$ & $0.0005(2)$ & $0.0153(2)$ & $0.0103(2)$ \\
\hline $\mathrm{Se}(1)$ & $8 f$ & $0.20158(5)$ & $0.05978(4)$ & $0.04569(8)$ & $0.0334(4)$ & $0.0367(4)$ & $0.0349(4)$ & $0.0098(3)$ & $0.0079(3)$ & $0.0037(3)$ \\
\hline $\operatorname{Se}(2)$ & $8 f$ & $0.38508(5)$ & $0.13056(4)$ & $0.52056(8)$ & $0.0413(5)$ & $0.0408(4)$ & $0.0351(4)$ & $-0.0077(3)$ & $0.0200(3)$ & $0.0051(3)$ \\
\hline $\mathrm{Se}(3)$ & $8 f$ & $0.16885(4)$ & $0.21614(4)$ & $-0.23361(7)$ & $0.0235(4)$ & $0.0357(4)$ & $0.0373(4)$ & $0.0008(3)$ & $0.0121(3)$ & $0.0046(3)$ \\
\hline $\operatorname{Se}(4)$ & $8 f$ & $0.04227(5)$ & $0.21696(4)$ & $0.02981(7)$ & $0.0342(4)$ & $0.0350(4)$ & $0.0288(4)$ & $0.0088(3)$ & $0.0116(3)$ & $0.0041(3)$ \\
\hline $\operatorname{Se}(5)$ & $4 e$ & 0 & $0.05131(6)$ & -1/4 & $0.0305(6)$ & $0.0262(5)$ & $0.0522(7)$ & 0 & $0.0020(5)$ & 0 \\
\hline $\operatorname{Se}(6)$ & $4 e$ & $1 / 2$ & $0.11960(5)$ & $1 / 4$ & $0.0374(6)$ & $0.0241(4)$ & $0.0292(5)$ & 0 & $0.0156(4)$ & 0 \\
\hline $\mathrm{Si}(1)$ & $8 f$ & $0.4370(1)$ & $0.2051(1)$ & $0.3791(2)$ & $0.026(1)$ & $0.0258(9)$ & $0.0253(9)$ & $-0.0015(7)$ & $0.0088(8)$ & $0.0001(7)$ \\
\hline $\mathrm{Si}(2)$ & $8 f$ & $0.1037(1)$ & $0.1330(1)$ & $-0.1034(2)$ & $0.024(1)$ & $0.0281(9)$ & $0.029(1)$ & $0.0027(7)$ & $0.0090(8)$ & $-0.0003(8)$ \\
\hline
\end{tabular}

\footnotetext{
* Correspondence author (e-mail: pkd@lamar.colostate.edu )
} 
Acknowledgments. This research was funded by the Department of Energy (grant no. DE-FG03-OZER15351. The authors acknowledge S. M. Miller for assistance in crystallographic topics.

\section{References}

1. Eisenmann, B.; Hansa, J.: Crystal structure of tetrasodium decaseienotetragermanate, $\mathrm{Na}_{4}\left[\mathrm{Ge}_{4} \mathrm{Se}_{10}\right]$. Z. Kristallogr. 205 (1993) 325-326.

2. Klepp, K. O.; Zeitlinger, M.: Crystal structure of tetracesium decasulfidotetragermanate, $\mathrm{Cs}_{4} \mathrm{Ge}_{4} \mathrm{~S}_{10}$. Z. Kristallogr. 215 (2000) 7-8.
3. Eisenmann, B.; Hansa, J.: Crystal structure of tetrapotassium decaselenidotetragermanate, $\mathrm{K}_{4}\left[\mathrm{Ge}_{4} \mathrm{Se}_{10}\right]$. Z. Kristallogr. 206 (1993) 101-102.

4. Wachhold, M.; Kanatzidis, M. G.: Surfactant-Templated Inorganic Lamellar and Non-Lamellar Hybrid Phases Containing Adamantane [GesSe 10] $]^{4-}$ Anions. Chem. Mater. 12 (2000) 2914-2932.

5. Sheldrick, G. M.: SHELXTL. Structure Determination Software Suite. V. 6.10. Bruker AXS, Madison, Wisconsin, USA 2000. 